\title{
The Role of PET/CT Imaging in Lung Cancer
}

\author{
David Ladrón de Guevara Hernández \\ Department of Radiology, Clinica Las Condes, Santiago, Chile \\ Email: $\underline{\text { dlg@clc.cl }}$
}

Received 30 June 2015; accepted 31 July 2015; published 3 August 2015

Copyright (C) 2015 by author and Scientific Research Publishing Inc.

This work is licensed under the Creative Commons Attribution International License (CC BY).

http://creativecommons.org/licenses/by/4.0/

(c) $\underset{\mathrm{EY}}{\mathrm{E}}$ Open Access

\begin{abstract}
Lung cancer presents a high prevalence around the world and constitutes the leading cause of death due to cancer in men and women. Proper staging is essential in order to provide the correct treatment and estimate survival. PET/CT imaging is the method of choice for the staging of lung cancer patients with a better performance than the traditional imaging methods. It allows defining the primary tumor more clearly, separating it from the atelectasis and neighboring fibrotic changes, thus achieving an improved $T$ evaluation while optimizing the target definition for radiotherapy. It presents greater sensibility and specificity than Computed Tomography (CT) when assessing the extra-thoracic and thoracic lymph nodes, modifying the $\mathrm{N}$ stage with regard to the traditional study in up to $25 \%$ of patients. PET/CT imaging is the method with the best performance for detecting extra-encephalic metastasis and for detecting unexpected lesions in up to $30 \%$ of patients. In order to achieve proper encephalic staging, a Magnetic Resonance Imaging scan must be performed or, a CT scan of the brain concomitant to the PET/CT. One to eight percent of lung cancer patients who are subjected to PET/CT imaging present a synchronous secondary extra pulmonary or pulmonary cancer when being diagnosed, with significant prognostic and therapeutic implications. PET/CT imaging has proven to be a valuable tool for survival prognosis with regard to the initial uptake of the tumor and the early response (interim) or final response to chemotherapy, although it is not routinely used for evaluating therapy. The PET/MRI is an emerging hybrid technique with certain advantages, such as less exposure to radiation in comparison to PET/CT and greater contrast resolution of the MRI images. However, its usefulness has not been validated yet for lung cancer patients.
\end{abstract}

Keywords

Lung Cancer, Pulmonary Malignancy, PET/CT

\section{Introduction}

Lung cancer is the leading cause of death due to cancer around the world, with 1.59 million deaths per year [1].

"Corresponding author. 
In spite of the progress achieved in treating these patients, survival after 5 years is still poor, with approximately $15 \%-16 \%$ [2] [3]. Tobacco is the main risk factor for lung cancer, increasing the likelihood of suffering this type of cancer 10 times more than a person who has not been exposed to it. Other conditions associated to a greater risk of lung cancer are idiopathic pulmonary fibrosis and expose to carcinogens such as asbestos.

Proper initial staging is fundamental for planning treatment and estimating the prognosis. PET (Positron Emission Tomography) imaging with F18-Fluorodeoxiglucose (F18-FDG) has been used in the initial study and staging of these patients, demonstrating advantages compared to traditional research. Since the year 2000, PET/CT (Computed Tomography) imaging has quickly been replacing PET imaging in clinical practice due to its functional-anatomical bimodal nature, which provides the exam with significantly greater diagnostic precision than just PET and CT analyzed separately [4]. Recent literature about the cost-effectiveness of PET/CT imaging has been mentioned as a recommendable option from an economic point of view [5].

\section{PET/CT}

PET/CT is a hybrid imaging technique that provides anatomical information of the CT and metabolic information of the PET, allowing to visualize both individually or fused in 3D or bidimensional slices. The most commonly used radiotracer is F18-FDG, which allows detecting primary tumors as well as metastasis that consume glucose, corresponding to the majority of the malignant pulmonary lesions. PET/CT imaging alone does not use iodine contrast agents, however, simultaneous acquisition of a complementary CT scan with contrast is a possibility in each exam and it is recommended when a recent CT scan of the thorax is unavailable. The use of iodined contrast improves visualization of the mediastinal vessels and its relation to the tumor, it helps evaluate the mediastinum and pleura, and allows to rule out concomitant pulmonary thromboembolism if the CT scan is carried out with angiographic technique.

\section{Staging of Lung Cancer}

The initial study of any lung cancer patient must be performed by a multi-disciplinary medical team that includes an expert radiologist, a nuclear medicine physician, an interventional radiologist, a pneumologist and a thoracic surgeon, using diagnostic tests which having high diagnostic accuracy and adequate cost-efficiency rate. Current treatment manuals recommend performing PET/CT imaging on any patient highly suspected to be suffering from lung cancer [3], preferably before histological studies are carried out since tissue samples must be useful not only to provide the histological diagnosis but to stage the disease as well. Therefore, in many cases it is preferable to perform a biopsy on a suspicious mediastinal adenopathy instead of the primary pulmonary tumor since it provides greater staging value (for example T2N2 with mediastinal biopsy guided by PET vs. T2NX without PET). On the other hand, for tumors associated to post-obstructive pneumonias and masses with extensive necrotic areas, PET/CT imaging provides valuable information for pinpointing the biopsy site (Figure 1).

85\% - 90\% of all lung cancers correspond to non-small cell (NSCLC), which includes: adenocarcinoma, squamous carcinoma and large cell carcinoma. Adenocarcinoma may or may not be associated to smoking and it usually manifests as a peripheral tumor with early distant metastasis. Its F18-FDG uptake tends to be less than the rest of the histological types, and some low uptake tumors can cause false negative results [6] [7].

Squamous carcinoma is closely linked to smoking and generally appears in the center of the lung. Large cell carcinoma usually corresponds to a large peripheral pulmonary mass with metastasis at the time of diagnosis. Squamous carcinoma as well as large cell carcinoma are F18-FDG avid. The NSCLC are staged in accordance with the tumor-node-metastasis (TNM) nomenclature which provides information on the stage of the disease thus allowing to guide the treatment and estimate the survival prognosis [8].

The remaining 10\% - 15\% corresponds to small cell lung cancer (SCLC) which presents a more aggressive biology and evolution with staging, treatment and prognosis that are different than the ones for NSCLC. This type of cancer is strongly linked to smoking. Its staging is different than for NSCLC and it is classified into two stages: limited, in which the disease is restricted to the ipsilateral hemithorax, including the mediastinum and ipsilateral supraclavicular, and extensive when the disease has surpassed the hemithorax [9]. Since it is an entirely different disease than NSCLC, the PET/CT imaging value in SCLC will be discussed in an especially dedicated chapter at the end of this article. 


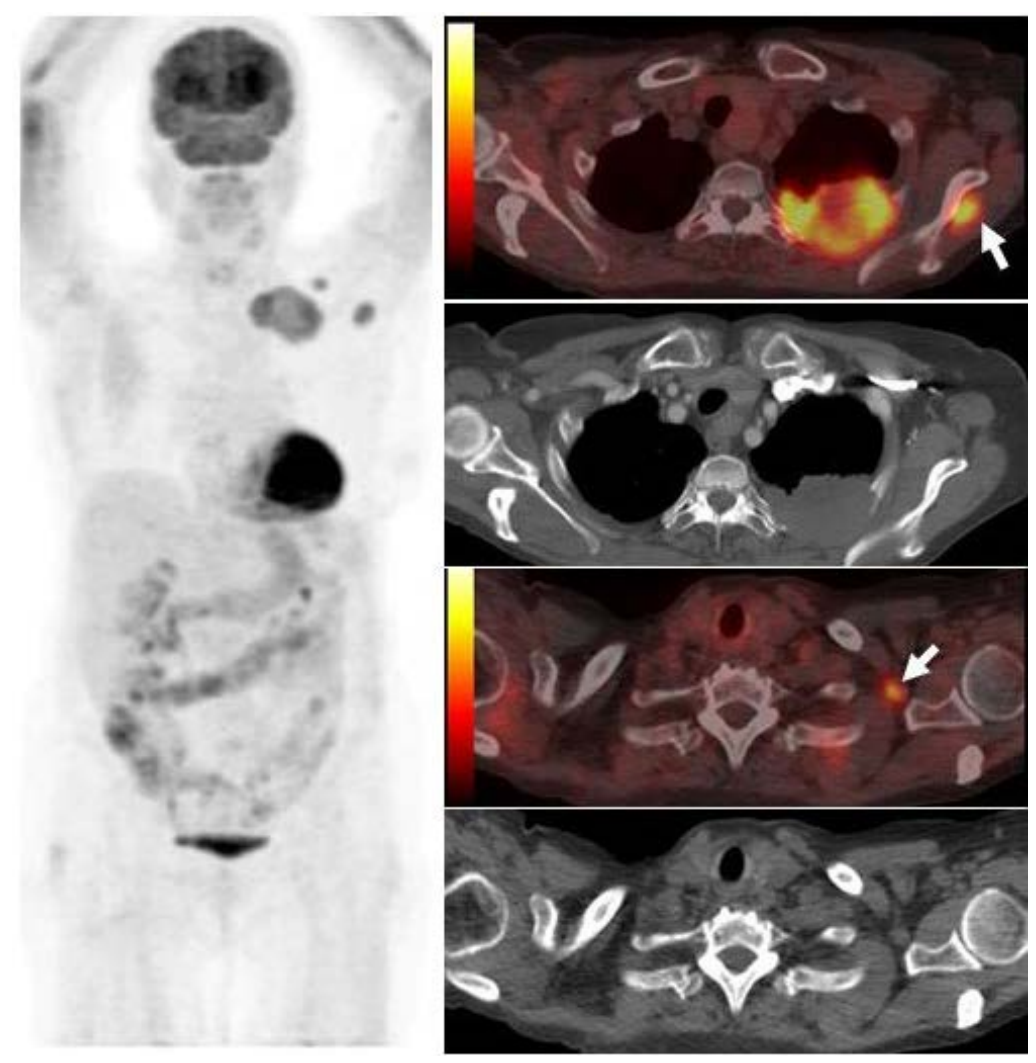

Figure 1. Partially necrotic left pulmonary tumor with rib involvement. Transthoracic needle biopsy has to be directed to periphery of tumor for viable sample. There are two metastases in infraclavicular node and muscular location (arrows), both of them negative in CT.

\subsection{T Staging}

The size of the tumor can be efficiently estimated through a CT scan in practically all cases. However, some tumors are associated to lung atelectasis phenomena or distal obstructive pneumonia which make estimating the size of the primary lesion more difficult. PET imaging allows to differentiate the atelectatic lung parenchyma of the malignant lesion through its F18-FDG uptake thus defining in a better manner the tumor. PET/CT imaging also helps to evaluate the invasion by the tumor into the pleura and mediastinum [10]. Pleural uptake is always pathological and within a neoplastic pulmonary context it is highly suggestive of a secondary origin. In tumors compromising the mediastinum, the concomitant acquisition of a CT scan with intravenous iodinated contrast is fundamental in order to study the relation of the tumor with the large blood vessels and pericardium.

The intensity of F18-FDG uptake by the primary pulmonary tumor is related to its biology and the degree of aggressiveness of the neoplastic cells, and can predict recurrence in the case of an elevated uptake. Kim et al. demonstrated in a cohort of patients who had been subjected to lung cancer surgery with postsurgical pathological N0 (pN0), that those who relapsed had a higher SUV ${ }_{\max }$ in lung primary tumor than the group without relapse, and they determined a cutoff of $S_{U V} V_{\max }$ of 6.9 in order to identify those patients with a higher risk of relapse [11]. They concluded that this higher risk subgroup could benefit from a more aggressive initial therapy.

\subsection{N Staging}

PET/CT imaging allows to metabolically characterize the hilar and mediastinal lymph nodes better than any other imaging technique since it manages to identify small hypermetabolic lymph nodes that are negative under CT morphological criteria as well as enlarged lymph nodes with no or little uptake that are frequently reactive or residual without neoplastic compromise [6]. In node staging, PET/CT imaging presents a sensitivity of $85 \%$ vs. $61 \%$ for CT and a specificity of $90 \%$ vs. $79 \%$ for CT [12]. 
The greatest advantage of PET/CT imaging in the mediastinum is its ability to rule out any lymph node compromise with a high degree of precision. A pulmonary tumor that is FDG avid with a metabolically negative mediastinum has a negative predictive value of 99\% [13]. However, not all lymph nodes with uptake are necessarily malignant and may correspond to false positives due to inflammation. This is the reason why the interpretation of the mediastinum must be complemented by FDG uptake indicators such as the SUV (Standardized Uptake Value), which improve their performance. $\mathrm{SUV}_{\max }$ cutoff values of 4.0 to 5.3 have been proposed as appropriate for considering lymph node uptake in the mediastinum as positive [7] [13] [14].

Proper metabolic mapping of mediastinal lymph nodes that allows to direct the transbronchial needle aspiration guided by ultrasonography can avoid a mediastinoscopy, particularly in areas that are difficult to access such as the aortopulmonary window and posterior subcarinal area [15].

The greatest contribution of PET/CT imaging in lymph node staging is in the early stages of the disease (I y II), where it produces a change in stage in up to $20 \%-25 \%$ of all cases thus avoiding unnecessary thoracotomies [16]. In a cohort of patients with lung cancer that included Stages I, II,III and IV, Fischer et al. found that for every 5 PET/CT imaging scans performed, one futile thoracotomy was avoided [4].

\subsection{Staging}

$40 \%-50 \%$ of lung cancer patients present metastasis when diagnosed [3] [7] [17]. The presence of distant metastasis radically modifies treatment of those patients, making the option of undergoing surgery much more difficult which in turn results in a poorer prognosis. PET/CT imaging presents undeniable advantages in the study of metastatic disease with regard to conventional imaging, particularly in lung cancer, which is characterized by presenting metastasis in multiple organs and frequently without a clearly defined order of dissemination. The high FDG uptake in lung cancer metastasis and the ability of PET/CT imaging to explore the entire body at once makes this the exam of choice for detecting extra-encephalic implants (NCCN). PET/CT imaging can detect unsuspected distant metastasis in up to $30 \%$ of all cases [15] [18].

The most common secondary lesions in lung cancer are bone lesions, present in $8 \%-27 \%$ of patients at the time of diagnosis [3] [6] [7] [19]. It is precisely with these types of lesions where the superiority of PET/CT imaging can be seen when compared to other imaging methods. Since more than half of bone metastisis involve only bone marrow without significant destruction of the bone structure, a radiography and CT scan are unable to detect them (Figure 2) and even a Tc-99m MDP bone scan has low sensitivity to this particular type of lesion. This also occurs with pure lytic bone lesions, which tend to be FDG avid but may not show increase of uptake in bone scan when they have no associated osteoblastic activity [6] [7] [17].

Abdominal metastases are common in lung cancer patients and they are predominantly located in the liver or adrenal glands [6]. Due to the high prevalence of hepatic and adrenal benign lesions in the adult population, it is necessary to have imaging tests that are able to recognize them and differentiate them from a metastasis. In the case of adrenal gland lesions, a moderate or high FDG uptake by the lesion has a high positive predictive value

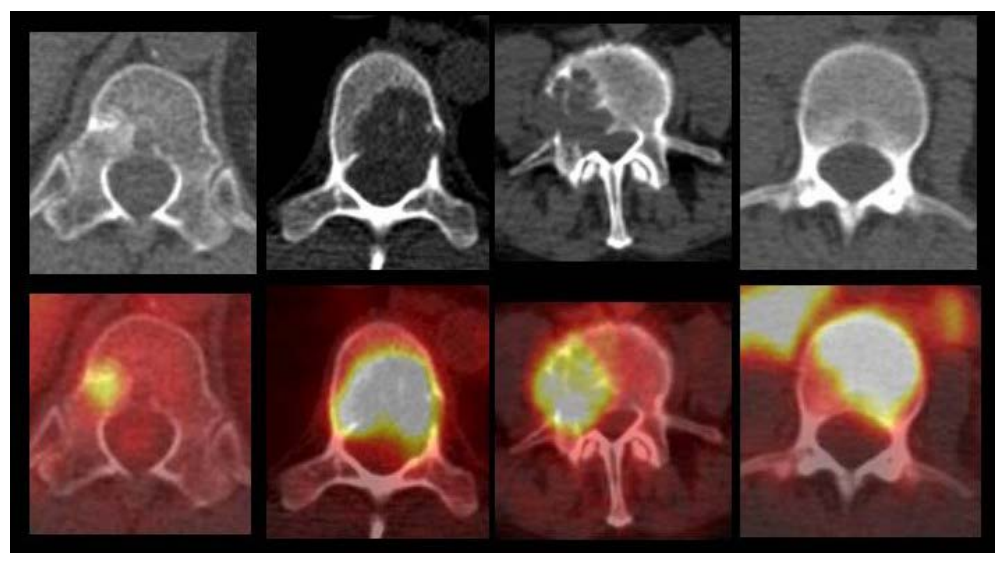

(a)

(b)

(c)

(d)

Figure 2. Types of bone metastases in lung cancer. (a) Blastic; (b) Lytic; (c) Mixed; (d) Bone marrow. 
for metastasis. In a meta-analysis of adrenal lesion characterization published by Boland et al., the sensitivity and specificity of PET using the SUV was $87 \%$ and $87 \%$ in the general population, and $94 \%$ and $82 \%$ in the lung cancer population respectively [20]. The best cutoff value of $\mathrm{SUV}_{\max }$ to differentiate malignant from benign lesions seems to be 2.5 [20] [21].

In the case of liver metastasis, practically all benign lesions (cysts, hemangiomas, focal nodular hyperplasia) show less or the same FDG uptake as the normal liver parenchyma. Any nodule or mass that demonstrates greater uptake that normal liver parenchyma is highly suspected of being malignant (Figure 3).

For adrenal, liver and bone metastases, a whole-body magnetic resonance imaging with diffusion-weighted (DW) imaging is a valid alternative when PET/CT imaging is unavailable [22].

PET/CT imaging allows to evaluate areas that are not covered by the conventional staging study, detecting occasional metastasis in skin, skeletal muscles, soft tissue, pancreas and kidneys [6] [7]. Although rare, said lesions can be important in the initial staging of patients with no apparent metastasis or with oligometastases since it allows to confirm the multifocal nature of the disease (Figure 1).

\subsection{Encephalic Staging}

Even though secondary brain lesions are more infrequent in this population, its importance lies in the fact that its presence results in a high morbidity and mortality rate, which in some cases can be avoided or at least diminished through proper treatment. Since the brain consumes high quantities of glucose in its basal state, and the metastatic lesions can be disguised by the high physiological uptake of the brain, PET sensitivity for detecting metastasis is limited. Due to the latter, current protocols recommend the additional use of Magnetic Resonance Imaging (MRI) in order to stage the brain as of stage IB [3]. In the case that an MRI is unavailable, it is possible to perform a brain CT with intravenous iodinated contrast when the PET/CT imaging is being carried out, with results quite similar to the brain MRI [23].

\section{Synchronous Pulmonary and Extra Pulmonary Malignancies}

1\% - 8\% of lung cancer patients present another synchronous cancer at the time of diagnosis [7] [24]. As in the case of lung cancer, most of these malignancies will be associated to tobacco. In fact, 6.5\% of chronic smoker patients who undergo staging for any type of cancer, will present another synchronous cancer at the time of diagnosis [25].

In the case of multiple lung cancer, the diagnostic criteria used are: 1) both pulmonary lesions have different histologies, 2) the histologies are the same but there is no lymph node involvement and no extrathoracic metastases [3]. Considering these criteria, the frequency of synchronous multiple lung cancer described in the literature is $0.5 \%-3.3 \%$ [26].

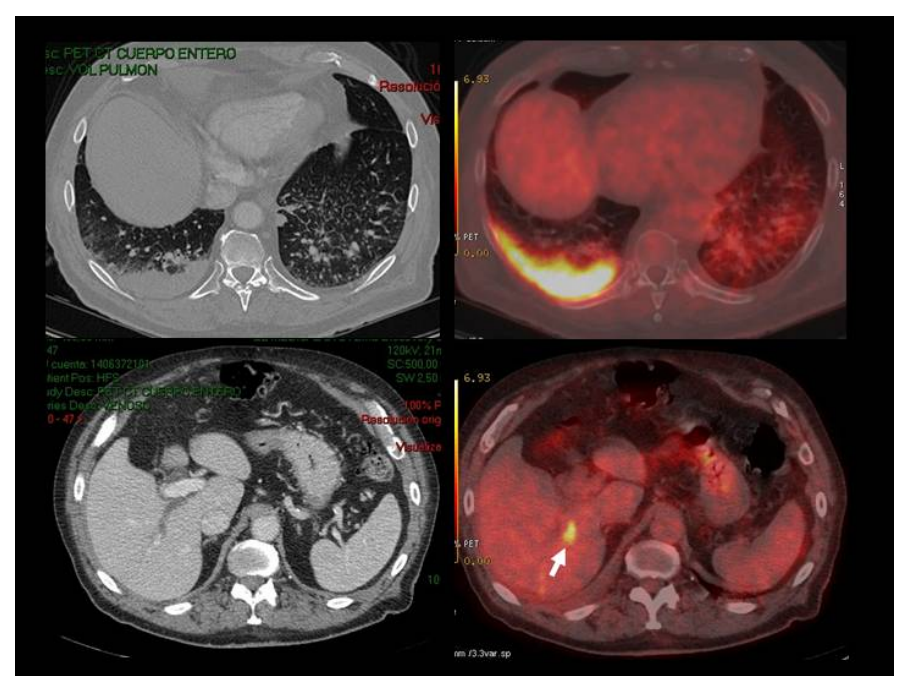

Figure 3. Right lung adenocarcinoma. There is one liver metastases (arrow) that is not visible in contrasted CT. 
The most common extra pulmonary locations of synchronous cancer detected by PET/CT imaging in lung cancer patients are the breasts, head and neck, esophagus and thyroid [7] [24] [25]. More than $80 \%$ of these synchronous lesions are detected in an early stage, which allow its curative treatment [7] [24]. However, given the nature of the population in question, the relevance of this finding is relative and strongly depends on how far the lung cancer has spread at the time of diagnosis.

\section{Radiotherapy Planning}

The ability of PET imaging to differentiate metabolically active tumoral tissue from atelectasis, post-obstructive pneumonia or fibrotic changes is crucial when seeking gross tumor volume (GTV) delineation, and it has a high impact on radiotherapy planning, modifying the form and size of the target volume, and decreasing exposure of the atelectatic lung parenchyma or other organs at risk (i.e. esophagus, heart, spinal cord) near the tumor [27]. Furthermore, it significantly modifies the mediastinal field since it detects more lesions than CT [6] [12]. With the incorporation of PET imaging in the traditional evaluation, changes in the target volume have been reported in more than $20 \%$ of patients, and modifications in the stage in $20 \%-50 \%$ of all cases. Diverse literature has described a change in Planning Target Volume (PTV) in more than 50\% of patients with NSCLC [27] [28]. The best method of GTV delineation has yet to be defined but it most likely corresponds to a combination of a visual evaluation with semi-quantitative methods based on SUV in which the most appropriate cutoff value seems to be a $\mathrm{SUV}_{\max }$ of 2.5. Given that said value may vary due to the size of the tumor and its histology, it is recom-

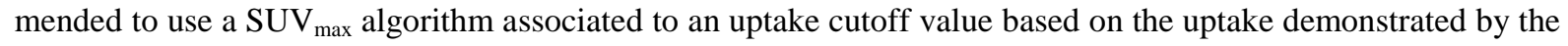
lesion [29]. GTV delineation can be optimized based on 4D-PET/CT images which minimize the changes in position of the lesions due to respiratory movements. This technique is particularly useful in image-guided radiation therapy (IGRT), and should be used whenever high doses are applied to the chest [27].

\section{Treatment Control and Prognosis}

The degree of dissemination at the time of diagnosis is the main prognosis factor in lung cancers subjected to surgical treatment, with a 5-years survival of $57 \%$ - 67\% in no metastatic disease (Stage I); $47 \%$ in the case of hilar lymph node involvement (Stage II), and 23\% in the case of ipsilateral mediastinal lymph node compromise (Stage IIIA). In the case of unresectable disease the survival rate is further decreased. The presence of distant metastasis is one of the main indicators of unresectability. In the case of oligometastasis detected with PET/CT imaging, the surgical option must be carefully evaluated especially if the metastasis is in a different lobe of the same lung (T4 in current classification). The precise staging provided by PET/CT imaging, and its ability to detect metastasis with a high degree of sensitivity makes it a prognostic tool per se when determining the TNM stage in an accurate and efficient manner.

In subgroups of patients that have a similar TNM stage, PET/CT imaging plays an additional prognostic role thanks to its ability to indirectly evaluate the biology of the tumor. FDG uptake by the tumor is different based on the biological nature of the neoplasia and the density of viable tumor cells. A direct relationship between the intensity of FDG uptake and the degree of neoplasia aggressiveness has been demonstrated [30]. The SUV value in lung tumors prior to treatment has shown a correlation with the survival rate in NSCLC [31]-[34]. Different SUV cutoff values have been described that result in prognostic differences ranging from 2.5 up to 20, depending on the SUV type under study ( $\mathrm{SUV}_{\max }, \mathrm{SUV}_{\text {mean }}$, etc.), the method of obtaining them (best cut-off, median, arbitrary), and different technical factors related to PET acquisition and SUV correction due to height/ weight. SUV $_{\max }$ values between 5 and 9 (corrected based on the weight of the patient) in primary tumors seems to be the most appropriate for differentiating patients with a better life expectancy (low SUV) and poorer life expectancy (high SUV) [31]-[34]. It is possible that the intrinsic prognostic value of SUV is overrated in the literature due to its strong association with other prognostic factors. Different authors have warned about the need to evaluate SUV as an independent factor, separating it properly from it others such as tumor size and the degree of dissemination of the disease [32].

The evaluation of treatment with PET/CT imaging has not been established as a routine practice in NSCLC. However, several authors have found certain usefulness in identifying Stage III patients with a favorable response to induction chemotherapy who had a better life expectancy, and who would be subjected to surgical treatment. Early metabolic response (interim PET) and late metabolic response (after completed the treatment) to chemotherapy has well demonstrated prognostic implications in lung cancers. As of the first cycle of chemo- 
therapy, survival curves can be drawn in accordance with the degree of decrease in glucidic metabolism in the tumor or in the mediastinal involvement, quantified using SUV [35].

\section{Surveillance}

Most literature does not support the use of systematic surveillance with images in patients treated for lung cancer, indicating that it is not cost-effective and it does not significantly modify the life expectancy of the patient [36]. Although early detection of recurrence improves the therapeutic options for the patient, there is controversy over the frequency and type of surveillance that should be applied.

In fact, PET/CT imaging has not an established role in lung cancer patients in post-treatment follow up and surveillance, in which clinical criteria tends to prevail and imaging tests are performed only on symptomatic patients or on those patients who are suspected of having relapsed. On the other hand, a chest CT is not routinely used during post therapy monitoring since most recurrences are not locoregional and correspond to distant metastasis [37]. Not even a brain MRI plays an established role in monitoring asymptomatic patients [37].

Accurate determination of the recurring disease allows to offer the patient reasonable curative or palliative care, whether it be surgery once again, chemotherapy or radiotherapy. It seems obvious that the earlier the recurrence is detected, the greater the number of therapeutic alternatives available and thus, the patient will have less morbidity. PET/CT imaging has proven to be the method with the greatest sensitivity and specificity in detecting post treatment recurrence, even more so than traditional methods [32]. The point in question is whether PET/CT imaging provides additional information that in turn substantially changes the treatment and management of these patients.

Hellwig et al. demonstrated the superiority of PET imaging in detecting recurrences and its ability to predict which patients would benefit from surgery again based on its level of uptake. They found significantly greater global survival in relapsed patients who showed SUV $<11$ [38], with regard to those patients presenting lesions with greater uptake.

Marcus et al. described that the fourth and subsequent 18F-FDG PET/CT scans performed during follow-up after completion of primary treatment detected recurrence in $44 \%$ of patients without clinical suspicion, and allowed to rule out recurrence in $24 \%$ under clinical suspicion, while $28 \%$ of patients were subjected to a change in their management [39].

When studying 100 NSCLC patients treated with curative intentions with radiotherapy, van Loon et al. demonstrated adequate cost-benefit of PET/CT imaging performed 3 months after treatment, with a better performance than a chest CT and the radiological/clinical evaluation [40]. Although PET/CT imaging turned out to be more expensive than the conventional follow up protocols, it allowed for significant savings of resources due to its better diagnostic performance, showing a greater probability of being cost-effective (73\%).

It seems that there is not sufficient evidence that allows to properly define the place of images and in particular, PET/CT imaging in the surveillance of patients treated for lung cancer. Said evidence should be focused on the cost-benefit rate of the different diagnostic methods and its adequate timing during the monitoring period.

\section{Small Cell Lung Cancer}

Small cell lung cancer corresponds to $10 \%$ - 15\% of all lung cancers and it is intrinsically linked to tobacco [9]. It is a different entity than NSCLC, more aggressive, frequently associated to metastasis at the time of diagnosis and with a generally poorer 5-years survival rate of approximately $1 \%-2 \%$. It is classified as a limited disease if the lesions are confined to the hemithorax or as an extensive disease if there is metastasis outside of the hemithorax. In these patients, surgery is limited only to cases of small tumors without compromise of the lymph nodes, and systemic treatment is applied in almost all cases, with or without associated radiotherapy [3].

$\mathrm{PET} / \mathrm{CT}$ imaging is useful in the initial evaluation, mainly in limited disease since it modifies the traditional staging in $10 \%-33 \%$ of all cases, with the corresponding change in management [15]. In SCLC, PET/CT imaging allows to detect extrathoracic metastasis in $10-20 \%$ of all patients with a negative conventional study, with an obvious impact on treatment [41]. As can be expected, due to its greater sensitivity, PET/CT imaging tends to increase the number of patients with extensive disease, although it also modifies staging in an inverse manner, meaning, it modifies the diagnosis of some patients from extensive disease to limited disease [42]. Due to the lower sensitivity of PET/CT imaging in the brain, a MRI must be used to evaluate the brain in all patients [3].

Interim post chemotherapy PET/CT imaging can identify patients who have not responded adequately to 
treatment thus avoiding unnecessary treatment. However, specifically in SCLC, there is not sufficient evidence to support this, and PET/CT imaging is not routinely used nor is it recommended in the current manuals for this effect.

Similarly to NSCLC, F18-FDG uptake of the tumor before treatment has an important survival prognostic value, with greater mortality seen in those lesions with a greater uptake. In a prospective study of 67 patients with SCLC, Lee et al. found a lower survival rate in patients with $\mathrm{SUV}_{\max } \geq 8.7$ (11.7 months, 95\% CI, 7.5 15.9 ) vs. $\mathrm{SUV}_{\max }<8.7$ (24.3 months, 95\% CI, 17.3 - 31.4). The cutoff value of $\mathrm{SUV}_{\max } 8.7$ also demonstrated significant prognostic value when separating the population into subgroups of limited disease (10.7 vs. 23.5 months) and extensive disease (9.5 vs. 17.7 months) [43]. This group also found an interesting prognostic value when combining the $\mathrm{SUV}_{\max }$ with the degree of dissemination of the disease, identifying a subgroup with low $\mathrm{SUV}_{\max }$ and limited disease that presented better life expectancy (31.1 months, 20.1 - 42.2), and another group with a high $\mathrm{SUV}_{\max }$ and extended disease which presented the higher mortality (9.8 months, 5.5 - 11.9).

\section{Value of PET/MRI Imaging in Lung Cancer}

The advent of PET/MRI imaging in clinical practice has shown certain comparative advantages with regard to PET/CT imaging, such as less exposure to radiation and the intrinsic characteristics of MRI images which include a high contrast resolution. With regard to PET/CT imaging, the new hybrid technique allows for an improved evaluation of the local invasion of the wall and/or mediastinum by the tumor, and provides greater sensitivity when studying the metastasis in the brain or liver [44]. However, its performance in detecting lung metastases is clearly poorer, with possible false negatives in particular with lesions measuring less than $5 \mathrm{~mm}$. This would limit not only $\mathrm{T}$ staging, with the possible omission of small nodes in the same lobe (T3) or same lung (T4), as well as M staging due to possible false negatives in the contralateral lung. An important point to consider is the quantification of PET uptake (SUV), of great importance when evaluating response to treatment, and which has still not been validated for the PET/MRI systems [44] [45]. Until this occurs, and the PET/MRI attenuation correction algorithms are not optimized, it shall not be possible to recommend its regular clinical use in lung cancer patients.

\section{Conclusion}

PET/CT imaging is the method of choice for the extra-encephalic staging of lung cancer patients with a better performance than the traditional imaging methods. It has high accuracy in $\mathrm{T}$ and especially $\mathrm{N}$ and $\mathrm{M}$ assessment, and it allows detecting second unsuspected synchronous cancers. In order to achieve proper encephalic staging, a brain MRI must be performed as of stage IB. The evaluation of treatment and surveillance with PET/CT imaging has not been established as a routine practice in lung cancer, and there is not yet sufficient evidence that allows properly defining the place of PET/CT imaging in this matter.

\section{Conflict of Interest}

The author declares no conflict of interest.

\section{References}

[1] GLOBOCAN (2012) Estimated Cancer Incidence, Mortality and Prevalence Worldwide in 2012. http://globocan.iarc.fr

[2] Howlander, N., Noone, A. and Krapcho, M. (2012) SEER Cancer Statistics Review, 1975-2009 (Vintage 2009 Population) Based on November 2001 SEER Data Submission. National Cancer Institute, Bethesda. http://seer.cancer.gov/statfacts/html/lungb.html

[3] (2015) National Comprehensive Cancer Network Guidelines. http://www.nccn.org/professionals/physician_gls/f_guidelines.asp\#site

[4] Fischer, B., Lassen, U., Mortensen, J., Larsen, S., Loft, A., Bertelsen, A., et al. (2009) Preoperative Staging of Lung Cancer with Combined PET-CT. New England Journal of Medicine, 2, 32-29. http://dx.doi.org/10.1056/NEJMoa0900043

[5] Schreyogg, J., Weller, J., Stargardt, T., et al. (2010) Cost-Effectiveness of Hybrid PET/CT for Staging of Non-Small Cell Lung Cancer. Journal of Nuclear Medicine, 51, 1668-1675. http://dx.doi.org/10.2967/jnumed.109.072090 
[6] Silvestri, G.A., Gould, M.K., Margolis, M.L., Tanoue, L.T., McCrory, D., Toloza, E., et al. (2007) Noninvasive Staging of Non-Small Cell Lung Cancer. ACCP Evidenced-Based Clinical Practice Guidelines (2nd Edition). Chest, 132, 178S-201S.

[7] Ladron de Guevara, D., Furnaro, F., Yévenes, S., Clavero, J.M., Piottante, A., Lazo, D., Rodríguez, P., Undurraga, A., Álvarez, M., Pefaur, R. and Pardo, C. (2015) Positron Emission Tomography/Computed Tomography for Lung Cancer Staging. Revista Médica de Chile, 143, 22-29.

[8] UyBico, S.J., Wu, C.C., Suh, R.D., Le, H.N., Brown, K., Krishnam, M.S., et al. (2010) Lung Cancer Staging Essentials: The New TNM Staging System and Potential Imaging Pitfalls. RadioGraphics, 30, 1163-1181. http://dx.doi.org/10.1148/rg.305095166

[9] Samson, D.J., Seidenfeld, J., Simon, G.R., Turrisi, A.T., Bonnell, C., Ziegler, K.M., et al. (2007) Evidence for Management of Small Cell Lung Cancer. ACCP Evidence-Based Clinical Practice Guidelines (2nd Edition). Chest, 132, 314S-323S.

[10] Lardinois, D., Weder, W., Hany, T.F., Kamel, E.M., Korom, S., Seifert, B., et al. (2003) Staging of Non-Small-Cell Lung Cancer with Integrated Positron-Emission Tomography and Computed Tomography. New England Journal of Medicine, 348, 2500-2507. http://dx.doi.org/10.1056/NEJMoa022136

[11] Kim, D.H., Son, S.H., Kim, C.Y., Hong, C.M., Oh, J.R., Song, B.I., et al. (2014) Prediction for Recurrence Using F-18 FDG PET/CT in Pathologic N0 Lung Adenocarcinoma after Curative Surgery. Annals of Surgical Oncology, 21, 589596. http://dx.doi.org/10.1245/s10434-013-3270-5

[12] Gould, M.K., Kuschner, W.G., Rydzak, C.E., Maclean, C.C., Demas, A.N., Shigemitsu, H., et al. (2003) Test Performance of Positron Emission Tomography and Computed Tomography for Mediastinal Staging in Patients with NonSmall-Cell Lung Cancer: A Meta-Analysis. Annals of Internal Medicine, 139, 879-892. http://dx.doi.org/10.7326/0003-4819-139-11-200311180-00013

[13] Enoch Lee, B., Redwine, J., Foster, C., Abella, E., Lown, T., Lau, D., et al. (2008) Mediastinoscopy Might Not Be Necessary in Patients with Non-Small Cell Lung Cancer with Mediastinal Lymph Nodes Having a Maximum Standardized Uptake Value of Less than 5.3. The Journal of Thoracic and Cardiovascular Surgery, 135, 615-619. http://dx.doi.org/10.1016/j.jtcvs.2007.09.029

[14] Bryant, A.S., Cerfolio, R.J., Klemm, K.M. and Ojha, B. (2006) Maximum Standard Uptake Value of Mediastinal Lymph Nodes on Integrated FDG PET-CT Predicts Pathology in Patients with Non-Small Cell Lung Cancer. The Annals of Thoracic Surgery, 82, 417-423. http://dx.doi.org/10.1016/j.athoracsur.2005.12.047

[15] Ambrosini, V., Nicolini, S., Caroli, P., Nanni, C., Massaro, A., Marzola, M.C., et al. (2012) PET/CT Imaging in Different Types of Lung Cancer: An Overview. European Journal of Radiology, 81, 988-1001. http://dx.doi.org/10.1016/j.ejrad.2011.03.020

[16] Viney, R.C., Boyer, M.J., King, M.T., Kenny, P.M., Pollicino, C.A., McLean, J.M., et al. (2004) Randomized Controlled Trial of the Role of Positron Emission Tomography in the Management of Stage I and II Non-Small-Cell Lung Cancer. Journal of Clinical Oncology, 22, 2357-2362. http://dx.doi.org/10.1200/JCO.2004.04.126

[17] Cheran, S.K., Herndon, J.E. and Patz, E.F. (2004) Comparison of Whole-Body FDG-PET to Bone Scan for Detection of Bone Metastases in Patients with a New Diagnosis of Lung Cancer. Lung Cancer, 44, 317-325. http://dx.doi.org/10.1016/j.lungcan.2003.11.008

[18] Seltzer, M.A., Yap, C.S., Silverman, D.H., Meta, J., Schiepers, C., Phelps, M.E., et al. (2002) The Impact of PET on the Management of Lung Cancer: The Referring Physician’s Perspective. Journal of Nuclear Medicine, 43, 752-756.

[19] Hess, K.R., Varadhachary, G.R., Taylor, S.H., Wei, W., Raber, M.N., Lenzi, R., et al. (2006) Metastatic Patterns in Adenocarcinoma. Cancer, 106, 1624-1633. http://dx.doi.org/10.1002/cncr.21778

[20] Boland, G.W.L., Dwamena, B.A., Sangwaiya, M.J., Goehler, A.G., Blake, M.A., Hahn, P.F., et al. (2011) Characterization of Adrenal Masses by Using FDG PET: A Systematic Review and Meta-Analysis of Diagnostic Test Performance. Radiology, 259, 117-126. http://dx.doi.org/10.1148/radiol.11100569

[21] Brady, M.J., Thomas, J., Wong, T.Z., Franklin, K.M., Ho, L.M. and Paulson, E.K. (2009) Adrenal Nodules at FDG PET/CT in Patients Known to Have or Suspected of Having Lung Cancer: A Proposal for an Efficient Diagnostic Algorithm. Radiology, 250, 523-530. http://dx.doi.org/10.1148/radiol.2502080219

[22] Ohno, Y., Koyama, H., Onishi, Y., Takenaka, D., Nogami, M., Yoshikawa, T., et al. (2008) Non-Small Cell Lung Cancer: Whole-Body MR Examination for M-Stage Assessment-Utility for Whole-Body Diffusion-weighted Imaging Compared with Integrated FDG PET/CT. Radiology, 248, 643-654. http://dx.doi.org/10.1148/radiol.2482072039

[23] Hendriks, L.E.L., Bootsma, G.P., de Ruysscher, D.K.M., Scheppers, N.A.M., Hofman, P.A.M., Brans, B.T., et al. (2013) Screening for Brain Metastases in Patients with Stage III Non-Small Cell Lung Cancer: Is There Additive Value of Magnetic Resonance Imaging above a Contrast-Enhanced Computed Tomography of the Brain? Lung Cancer, 80, 293-297. http://dx.doi.org/10.1016/j.lungcan.2013.02.006 
[24] Lardinois, D., Weder, W., Roudas, M., von Schulthess, G.K., Tutic, M., Moch, H., et al. (2005) Etiology of Solitary Extrapulmonary Positron Emission Tomography and Computed Tomography Findings in Patients with Lung Cancer. Journal of Clinical Oncology, 23, 6846-6853. http://dx.doi.org/10.1200/JCO.2005.10.116

[25] Choi, J.Y., Lee, K.S., Kwon, O.J., Shim, Y.M., Baek, C.-H., Park, K., et al. (2005) Improved Detection of Second Primary Cancer Using Integrated $\left[{ }^{18} \mathrm{~F}\right]$ Fluorodeoxyglucose Positron Emission Tomography and Computed Tomography for Initial Tumor Staging. Journal of Clinical Oncology, 23, 7654-7659. http://dx.doi.org/10.1200/JCO.2005.01.4340

[26] Xue, X., Xue, Q., Wang, N., Zhang, L., Guo, L., Li, X., et al. (2012) Early Clinical Diagnosis of Synchronous Multiple Primary Lung Cancer. Oncology Letters, 3, 234-237.

[27] Chi, A. and Nguyen, N.P. (2014) The Utility of Positron Emission Tomography in the Treatment Planning of Image-Guided Radiotherapy for Non-Small Cell Lung Cancer. Frontiers in Oncology, 4, Article No. 273. http://dx.doi.org/10.3389/fonc.2014.00273

[28] Bradley, J., Thorstad, W.L., Mutic, S., Miller, T.R., Dehdashti, F., Siegel, B.A., et al. (2004) Impact of FDG-PET on Radiation Therapy Volume Delineation in Non-Small-Cell Lung Cancer. International Journal of Radiation Oncology, Biology, Physics, 59, 78-86. http://dx.doi.org/10.1016/j.ijrobp.2003.10.044

[29] Black, Q.C., Grills, I.S., Kestin, L.L., Wong, C.Y., Wong, J.W., Martinez, A.A., et al. (2004) Defining a Radiotherapy Target with Positron Emission Tomography. International Journal of Radiation Oncology, Biology, Physics, 60, 12721282. http://dx.doi.org/10.1016/j.ijrobp.2004.06.254

[30] Berghmans, T., Dusart, M., Paesmans, M., Hossein-Foucher, C., Buvat, I., Castaigne, C., et al. (2008) Primary Tumor Standardized Uptake Value $\left(\mathrm{SUV}_{\max }\right.$ ) Measured on Fluorodeoxyglucose Positron Emission Tomography (FDG-PET) Is of Prognostic Value for Survival in Non-Small Cell Lung Cancer (NSCLC): A Systematic Review and Meta-Analysis (MA) by the European Lung Cancer Working Party for the IASLC Lung Cancer Staging Project. Journal of Thoracic Oncology, 3, 6-12. http://dx.doi.org/10.1097/JTO.0b013e31815e6d6b

[31] Goodgame, B., Pillot, G.A., Yang, Z., Shriki, J., Meyers, B.F., Zoole, J., Gao, F., Dehdashti, F., Patterson, A., Siegel, B.A., et al. (2008) Prognostic Value of Preoperative Positron Emission Tomography in Resected Stage I Non-Small Cell Lung Cancer. Journal of Thoracic Oncology, 3, 130-134. http://dx.doi.org/10.1097/JTO.0b013e318160c122

[32] Erasmus, J.J., Rohren, E. and Swisher, S.G. (2009) Prognosis and Reevaluation of Lung Cancer by Positron Emission Tomography Imaging. Proceedings of the American Thoracic Society, 6, 171-179. http://dx.doi.org/10.1513/pats.200806-059LC

[33] Downey, R.J., Akhurst, T., Gonen, M., Vincent, A., Bains, M.S. and Larson, S. (2004) Preoperative F-18 Fluorodeoxyglucose-Positron Emission Tomography Maximal Standardized Uptake Value Predicts Survival after Lung Cancer Resection. Journal of Clinical Oncology, 22, 3255-3260. http://dx.doi.org/10.1200/JCO.2004.11.109

[34] Ladrón de Guevara, D., Fuentes, A., Fariña, C., Corral, C. and Pefaur, R. (2013) Prognostic Value of PET/CT in Lung Cancer. Retrospective Analysis of 47 Patients. Revista Méica de Chile, 141, 41-48. http://dx.doi.org/10.4067/S0034-98872013000100006

[35] Hoekstra, C.J., Stroobants, S.G., Smit, E.F., Vansteenkiste, J., van Tinteren, H., Postmus, P.E., et al. (2005) Prognostic Relevance of Response Evaluation Using $\left[{ }^{18} \mathrm{~F}\right]-2-$ Fluoro-2-Deoxy-D-Glucose Positron Emission Tomography in Patients With Locally Advanced Non-Small-Cell Lung Cancer. Journal of Clinical Oncology, 23, 8362-8370. http://dx.doi.org/10.1200/JCO.2005.01.1189

[36] Tu-Quynh, H.E., Yong-Fang, K. and Gulshan, S. (2013) Surveillance Following Lung Cancer Resection. Clinical Research in Pulmonology, 1, 1001-1008.

[37] Sudarski, S., Henzler, T. and Schoenberg, S.O. (2013) Post-Therapeutic Positron Emission Tomography/Computed Tomography for Early Detection of Non-Small Cell Lung Cancer Recurrence. Translational Lung Cancer Research, 2, 295-303.

[38] Hellwig, D., Groschel, A., Graeter, T.P., Hellwig, A.P., Nestle, U., Schafers, H.J., et al. (2006) Diagnostic Performance and Prognostic Impact of FDG-PET in Suspected Recurrence of Surgically Treated Non-Small Cell Lung Cancer. European Journal of Nuclear Medicine and Molecular Imaging, 33, 13-21. http://dx.doi.org/10.1007/s00259-005-1919-4

[39] Marcus, C., Paidpally, V., Antoniou, A., Zaheer, A., Wahl, R. and Subramaniam, R.M. (2015) ${ }^{18}$ F-FDG PET/CT and Lung Cancer: Value of Fourth and Subsequent Posttherapy Follow-Up Scans for Patient Management. Journal of Nuclear Medicine, 56, 204-208. http://dx.doi.org/10.2967/jnumed.114.147884

[40] van Loon, J., Grutters, J.P., Wanders, R., Boersma, L., Dingemans, A.M., Bootsma, G., et al. (2010) 18FDG-PET-CT in the Follow-Up of Non-Small Cell Lung Cancer Patients after Radical Radiotherapy with or without Chemotherapy: An Economic Evaluation. European Journal of Cancer, 46, 110-119. http://dx.doi.org/10.1016/j.ejca.2009.10.028

[41] Goerres, G.W., von Schulthess, G.K. and Steinert, H.C. (2004) Why Most PET of Lung and Head-and-Neck Cancer Will Be PET/CT. Journal of Nuclear Medicine, 45, 66S-71S. 
[42] Brink, I., Schumacher, T., Mix, M., Ruhland, S., Stoelben, E., Digel, W., et al. (2004) Impact of $\left[{ }^{18}\right.$ F]FDG-PET on the Primary Staging of Small-Cell Lung Cancer. European Journal of Nuclear Medicine and Molecular Imaging, 31, 1614-1620. http://dx.doi.org/10.1007/s00259-004-1606-X

[43] Lee, Y.J., Cho, A., Cho, B.C., Yun, M., Kim, S.K., Chang, J., et al. (2009) High Tumor Metabolic Activity as Measured by Fluorodeoxyglucose Positron Emission Tomography Is Associated with Poor Prognosis in Limited and Extensive Stage Small-Cell Lung Cancer. Clinical Cancer Research, 15, 2426-2432. http://dx.doi.org/10.1158/1078-0432.CCR-08-2258

[44] Yoon, S.H., Goo, J.M., Lee, S.M., Park, C.M., Seo, H.J. and Cheon, G.J. (2014) Positron Emission Tomography/ Magnetic Resonance Imaging Evaluation of Lung Cancer Current Status and Future Prospects. Journal of Thoracic Imaging, 29, 4-16. http://dx.doi.org/10.1097/RTI.0000000000000062

[45] Yankeelov, T.E., Peterson, T.E., Abramson, R.G., Garcia-Izquierdo, D., Arlinghaus, L.R., Li, X., Atuegwu, N.C., et al. (2012) Simultaneous PET-MRI in Oncology: A Solution Looking for a Problem? Magnetic Resonance Imaging, 30, 1342-1356. http://dx.doi.org/10.1016/j.mri.2012.06.001

\section{Abbreviations}

PET: Positron Emission Tomography

CT: Computed Tomography

MRI: Magnetic Resonance Imaging

F18-FDG: F18-Fluorodeoxiglucose

NSCLC: Non-Small Cell Lung Cancer

SCLC: Small Cell Lung Cancer

SUV: Standardized Uptake Value

GTV: Gross Tumor Volume

PTV: Planning Target Volume

IGRT: Image-Guided Radiation Therapy 